\section{Change in relations}

\section{Brian Mathew}

The Families of the Monocotyledons: Structure, Evolution and Taxonomy. By R.M.T. Dahlgren, H.T. Clifford and P. F. Yeo. Springer-Verlag:1985. Pp.520. DM294, \$103.20.

IT HAS long been acknowledged by students of the monocotyledonous section of the flowering plant kingdom that the classification, especially at family level, is very unsatisfactory. One can pick several areas where obviously fairly unrelated groups have been lumped together into a heterogeneous mass and the family label stuck upon it; in particular the Liliaceae comes to mind where, in the traditional sense, every petaloid monocotyledon with six stamens and a superior ovary was deposited. True lilies thus shared the same family bed as asparagus, hyacinths and yucca, to mention but a few of the more well-known and very divergent groups.

Although the recognition of smaller, more homogeneous families is not something new (Hyacinthaceae for example dates from 1802), the present study makes use of a wide range of modern data to give convincing explanations for their acceptance. The proposed new classification is, to say the least, alarming at first sight since some genera which were formerly placed

\section{THE ELEMENTS OF GRAPHING DATA William S. Cleveland}

Presents fundamental principles of graphical perception, describes principles that enhance graphical communication, and presents a wide variety of modern graphical methods useful for data analysis. Principles illustrated by many fascinating sets of data drawn from diverse areas of science and technology. Whether you use graphs for data analysis-or wish to communicate data to others-you will find that the graphical methods and principles are powerful tools that will increase your effectiveness in analyzing and reporting data.

comtenus: 1. Introduction 2. Principles of Graph Construction 3. Graphical Methods 4. Graphical Perception

July 1985. 336 pp. cloth \$27.95, paper \$18.95. Add sales tax for CA, KY, MA, MI, NC, NJ, NY, WA Monterey, CA 93940 together in one family are now in some instances separated at order level and given family status of their own. A notable "split" which will surprise many botanists is between the Order Asparagales and the Liliales; Asparagales contains not only Asparagus but also many of the former members of the Liliaceae and Amaryllidaceae (both in Liliales). The decision to recognize the Asparagales as a group is strongly influenced by the presence of the blackish carbon-based compound phytomelan in the seed epidermis; this however is not the sole basis for its recognition, and as one works through this extremely meaty tome it is clear that the authors have studied character states from many disciplines and used them with much discretion in forming their suggested classification.

There are excellent line drawings illus-

trating many of the recognized plant families and there are also some interesting phylogenetical diagrams. Keys to the families are provided, but although these are particularly helpful it is perhaps optimistic of the authors to think that they are practical enough to be useful "in the field" when, for example, one of the dichotomies in the key to the Asparagales includes "Chromosome complement strongly dimorphic" versus "Chromosomes more uniform in size".

There are still many ifs, buts and maybe's. But this study represents one of the major steps forward in the understanding of the present relationships and the evolutionary pathways in the monocotyledons.

Brian Mathew is a Principal Scientific Officer in the Herbarium, Royal Botanic Gardens, Kew, Richmond, Surrey TW9 $3 A B, U K$.

\section{The light fantastic}

\section{Ann Wintle}

Thermoluminescence of Solids. By S.W.S McKeever. Cambridge University Press: 1985. Pp.376. £40, \$69.50.

OVEr the past 30 years people from a variety of different disciplines have attempted to use thermoluminescence (TL) as a research tool. The basis of the method is the ability of a large number of insulators and semiconductors to produce a measurable light signal when they are heated after exposure to radiation. Although there had been many observations of TL in naturally occurring minerals before the Second World War, it was not until the 1950s that several possible applications were seriously considered. Since then the technique has been used, over-extended and occasionally misused. In this book McKeever has brought together for the first time accounts of all the various uses of TL.

Research into applications as diverse as measurement of radiation doses in the natural environment and in medical treatment, dating of pottery from archaeological sites and the characterization of meteorites is carried out by different groups of physicists; they hardly ever attend the same conferences, or even read the same journals, and yet their research has the same basis and they use the same analytical procedures. McKeever states that his book is intended as a step "towards the unification of the insular approaches by presenting the topic of thermoluminescence as a single subject". I think he succeeds.

The book is fluently written, wellproduced, clearly laid out in nine chapters and includes over 1,000 references, most of them from the past ten years. Three of the first four chapters provide the historical and theoretical background on which the applications described in the remaining chapters rely. Chapter 3 deals with the experimental determination of the basic parameters which characterize the shapes of the glow curves, and which are used to predict the stability of TL signals either for dosimetry measurements or dating. Chapter 6 provides a comprehensive review of TL phosphors and their use in environmental monitoring, personal dosimetry and medical applications. This includes discussion of the responses to different types of radiation. The subsequent chapter covers the use of naturally occurring dosimeters for dating heated archacological materials and the recent extension to dating unheated sedimentary deposits. This empirical approach contrasts with studies on alkali halides and quartz for which TL is just one of the methods for characterizing the defect structure.

The complexity of the TL properties of geological materials is exemplified in a further chapter which deals with meteoritic and lunar samples as well as terrestrial ones. In particular, stony meteorites have been the subject of a great deal of work since they are potentially the source of a considerable amount of information, and McKeever critically reviews the relevant literature. It is clear that for all natural matter the experimental TL worker is at the mercy of nature; however, detailed studies of the basic TL properties of the individual minerals can provide useful information on the overall thermal, optical and radiation histories of such materials.

The technical nature of the book does not detract from its readability and it provides an excellent, critical introduction to the subject for graduate students. With its extensive bibliography, it will also be a helpful work of reference for anyone with more than a passing interest in the diverse fields of applied thermoluminescence.

Ann Wintle is at the Godwin Laboratory, University of Cambridge, Free School Lane, Cambridge CB2 $3 R S$, UK. 